\title{
Temporal diagnostics of femtosecond electron bunches with complex structures using sparsity-based algorithm
}

\author{
Q. Q. Su, J. F. Hua, ${ }^{*}$ Z. Nie, Y. Ma, S. Liu, Y.F. Zheng, C.-H. Pai, and W. Lu \\ Department of Engineering Physics, Tsinghua University, Beijing 100084, China
}

(Received 24 January 2018; published 2 November 2018)

\begin{abstract}
Femtosecond electron bunches with complex temporal structures play a crucial role in terahertz generation, free-electron lasers, and plasma wakefield accelerators. However, diagnostics of electron beam current profiles with femtosecond resolution are still challenging. Coherent transition radiation (CTR) has been proven to have the capability to reconstruct current profiles based on prior knowledge or complete information of spectra. In this paper, a weighted greedy sparse phase retrieval (WGESPAR) algorithm is developed to reduce the ambiguities during reconstruction of complex current profiles. This algorithm can achieve better performance than the Kramers-Kronig method and the Bubblewrap algorithm in the case of reconstructing complex temporal structures from truncated noisy CTR spectra. Based on the WGESPAR algorithm, the complex temporal structures of femtosecond electron bunches generated from laser wakefield accelerators can be successfully reconstructed.
\end{abstract}

DOI: 10.1103/PhysRevAccelBeams.21.112801

\section{INTRODUCTION}

Femtosecond (fs) electron beams have widespread applications in plasma-based accelerators, ultrafast electron microscopy, compact ultrafast $\mathrm{x}$-ray sources such as freeelectron lasers, and Compton scattering sources [1-6]. The temporal diagnostics of few-fs electron bunch profiles hence have become crucial in enhancing the performance of these facilities.

Methods of picosecond and subpicosecond electron bunch length measurements, such as deflecting cavities [7], electro-optic techniques [8], and frequency-domain methods $[9,10]$, have been well developed for conventional accelerators. However, the temporal diagnostics of few-fs electron bunches cannot directly utilize these methods due to their limited resolutions. Since the amplitude of the transverse deflecting voltage is one of the critical constraining factors, deflecting cavities are very difficult to reach the desired fs resolution. Recently, ultrashort lasers or terahertz (THz) pulses have been introduced to achieve subfs resolution [11-14]. For electro-optic techniques, the resolution is strongly influenced by the crystal response function [15], and the achievable temporal resolution has been demonstrated down to a few tens of femtoseconds. Frequency-domain methods have the potential to achieve sub-fs resolution by measuring the autocorrelation curve of

\footnotetext{
*jfhua@tsinghua.edu.cn
}

Published by the American Physical Society under the terms of the Creative Commons Attribution 4.0 International license. Further distribution of this work must maintain attribution to the author(s) and the published article's title, journal citation, and DOI. coherent transition radiation (CTR), coherent diffraction radiation, or coherent synchrotron radiation $[10,16,17]$.

To measure the durations of few-fs electron bunches, the method of CTR has been widely adopted. CTR is generated when electron bunches propagate through inhomogeneous media, and the beam current profile can be reconstructed from the attained CTR spectrum combined with the retrieved phase or based on the fitting of Gaussian bunches [18-20]. The Kramers-Kronig (KK) method is usually utilized to retrieve the phase from the CTR spectrum in conventional accelerators [21,22], but an extrapolation to a low frequency will bring unavoidable uncertainty for complex temporal structures [23]. Iterative algorithms such as the Bubblewrap algorithm have been developed for the temporal characterization of fs electron bunches, and a twobunch structure can be reconstructed $[24,25]$. However, its performance on complex current profiles from a noisy spectrum has not been mentioned. Recently, a greedy sparse phase retrieval (GESPAR) algorithm has been applied in coherent diffraction imaging and achieves good performance with prior knowledge [26,27]. The GESPAR algorithm can be heuristically applied in the reconstruction of current profiles from CTR spectra.

In this paper, a weighted greedy sparse phase retrieval (WGESPAR) algorithm is developed to reconstruct the complex temporal profiles of few-fs electron bunches based on truncated noisy spectra. In Sec. II, the theory of CTR diagnostics is briefly described. In Sec. III, a WGESPAR algorithm is developed for the reconstruction of complex current profiles from truncated noisy spectra; the algorithm's performance is compared with the KK method and the Bubblewrap algorithm. The effectiveness of the WGESPAR algorithm is confirmed by the synthetic 
simulation of the OSIRIS particle-in-cell (PIC) code and Monte Carlo code. Conclusions are drawn in Sec. IV.

\section{THEORY OF CTR DIAGNOSTICS}

Transition radiation is emitted when charged particles pass through inhomogeneous media. In the transverse dimensionless case, the CTR energy spectrum density $\frac{d I}{d \omega d \Omega}$ observed in the solid angle of $d \Omega$ is given by

$$
\frac{d I}{d \omega d \Omega}=N^{2}|\hat{\rho}(\omega)|^{2} \frac{d I_{e}}{d \omega d \Omega} .
$$

Here, $N$ denotes the total number of electrons, and the $\hat{\rho}(\omega)$ is the Fourier transform of the electron current profile. Specifically, the form factor of a Gaussian beam is $e^{-\frac{\sigma_{t}^{2} \omega^{2}}{2}}$, where $\sigma_{t}$ is the bunch duration. $\frac{d I_{e}}{d \omega d \Omega}$ means the spatial energy distribution of the CTR generated by a single electron. Neglecting the diffraction effect of the radiator, the spatial energy distribution of a single electron can be approximated as [28]

$$
\frac{d I_{e}}{d \omega d \Omega}=\frac{e^{2}}{2 \pi^{2} c}\left(\frac{\vec{\beta} \times \hat{n}}{1-\hat{n} \cdot \vec{\beta}}-\frac{\vec{\beta}^{\prime} \times \hat{n}}{1-\hat{n} \cdot \vec{\beta}^{\prime}}\right),
$$

where $\vec{\beta}$ and $\overrightarrow{\beta^{\prime}}$ denote the normalized velocity of the electron and its image charge, respectively, and $\hat{n}$ is the unit vector pointed toward the observer. According to Eqs. (1) and (2), the beam current profile is related only to $\hat{\rho}(\omega)$. Hence, one can reconstruct the current profile from the measured spectrum.

The reconstruction of beam currents from CTR spectra can be treated as one-dimensional phase retrieval problems, which have been studied for decades. The process can be summarized as seeking a current profile $\rho(t)$, the Fourier transform $\hat{\rho}(\omega)=\int_{-\infty}^{+\infty} \rho(t) \exp (-j \omega t) d t$ of which has the same amplitude as the measured CTR spectrum $|\hat{g}(\omega)|$. During the reconstruction, the missing phase information is expected to be retrieved from the modulus of CTR spectrum $|\hat{g}(\omega)|$. For physical considerations, the function $\rho(t)$ must be non-negative and constrained in a finite support.

For bunch length measurements in the picosecond region, the spectrum is obtained by scanning the autocorrelation curve from well-designed spectrometers to ensure an unambiguous reconstruction, while single-shot measurements are necessary for fs electron beams generated from laser plasma accelerators. During the single-shot measurement, the spectrum is usually truncated due to the sensitivity response of the spectrometer and has unavoidable noise. Hence, both the amplitude and the phase should be extrapolated to the unmeasured frequency, leading to unavoidable ambiguities when the extrapolation is inaccurate. Therefore, appropriate reconstruction algorithms should be developed for such applications.

\section{ALGORITHMS FOR LONGITUDINAL BUNCH PROFILE RECONSTRUCTION}

Several algorithms such as the KK method [21] and iterative algorithms, including the Gerchberg-Saxton (GS) algorithm [29] and the hybrid input-output (HIO) algorithm [30], have been developed to solve the phase retrieval problem. Iterative algorithms can reconstruct beam currents with sufficient information in the frequency domain, but they may lead to longer bunch tails if low-frequency information is missed. Based on the GS and HIO algorithms, the emergent Bubblewrap algorithm can alleviate this problem by adaptive support selection. Ambiguities in onedimensional phase retrieval problems have been a challenging issue for decades. By means of iterative algorithms and cross-correlation analysis, the two-step method has resolved the time translation and time reversal ambiguities and successfully reconstructed complex beams with long tails [23]. With the assumption that the function is restricted to a finite interval $[-a, a]$, the "zero-flipping" ambiguity has been introduced as a "nontrivial" ambiguity other than the known time translation and time reversal ambiguities [31]. Recently, it has been proven that one-dimensional phase retrieval problems still have ambiguities even with the assumption of non-negativity [32]. With the development of compressed sensing in the past few years, sparsity-based algorithms have been widely applied in the signal processing field for their hyperresolution and robustness even with highly noisy spectra. It has been discovered that sparsity can guarantee uniqueness in phase retrieval problems [33]. Accordingly, optimization-based algorithms, such as semidefinite programming [34], the GESPAR algorithm [26], and the sparse Fienup algorithm [35], have been proposed to solve phase retrieval problems with the sparsity priori, that is, assuming that the signal is sparse with a specific representation. In recent years, the GESPAR algorithm has been adopted in coherent diffraction imaging and made a good performance with sparsity as prior knowledge [27]. In this section, we develop a WGESPAR algorithm for current profile reconstruction and compare its performance with the KK and Bubblewrap algorithms.

\section{A. One-dimensional phase retrieval algorithms}

The KK method has been widely used to reconstruct current profiles from CTR spectra in traditional accelerators. The Fourier transform of the current profile $\rho(t)$ is given by $\hat{\rho}(\omega)=|\hat{g}(\omega)| e^{j \phi(\omega)}$. Here, $\rho(t)$ is a real, nonnegative signal with a certain support, and $|\hat{g}(\omega)|$ is the square root of the band-limited spectrum measured in the experiment. The approximate minimal phase $\phi(\omega)$ can be retrieved directly from the measured spectrum amplitude $|\hat{g}(\omega)|$ by the KK correlation [21] 


$$
\phi(\omega)=-\frac{2 \omega}{\pi} \int_{0}^{\infty} d x \frac{\ln [\rho(x) / \hat{\rho}(\omega)]}{x^{2}-\omega^{2}} .
$$

The extensively used GS and HIO algorithms are errorreducing algorithms, which achieve a better approximation at each iteration. A random function $\rho_{0}(t)$ can act as the initial beam current profile. The $k$ th trial solution $\rho_{k}(t)$ is Fourier transformed, yielding $\left|\hat{\rho}_{k}(\omega)\right| e^{j \phi_{k}(\omega)}$. To satisfy the Fourier-domain constraints, a better estimation of $\hat{\rho}(\omega)$ is given by $\hat{\rho}_{k}^{\prime}(\omega)=|\hat{g}(\omega)| e^{j \phi_{k}(\omega)}$. Then, the resulting $\hat{\rho}_{k}^{\prime}(\omega)$ is inverse Fourier transformed, yielding the function $\rho_{k}^{\prime}(t)$. $\rho_{k}^{\prime}(t)$ is then modified to satisfy the time-domain constraints, yielding $\rho_{k+1}(t)$, which is regarded as the input of the next iteration. Here, the Fourier-domain constraints come from the measured CTR spectrum, and the timedomain constraints are non-negativity and finite support. Normally, a relaxation factor $r$ is introduced to improve performance. This relaxation iterative format is called the HIO algorithm. When $r=-1$, the HIO algorithm simplifies to the GS algorithm. As an iterative algorithm stemming from the GS and HIO algorithms, the Bubblewrap improves its performance by adaptive support selection. At each iteration, a threshold is applied to the reconstructed signal to adaptively modify the support, alleviating the problem of the HIO and GS algorithms with large support.

In iterative algorithms, such as GS and HIO, overfitting usually occurs, leading to unphysical results when some information is missing or the measured spectra are highly noisy. From a different perspective, the GESPAR algorithm considers the phase retrieval as a nonlinear least-squares optimization problem, therein minimizing the squared distance $E=\left\|\hat{\boldsymbol{\rho}}^{2}-|\hat{\boldsymbol{g}}|^{2}\right\|^{2}$ between the square of the discrete Fourier transform of the reconstructed signals $\hat{\boldsymbol{\rho}}^{2}$ and the measured spectrum $|\hat{\boldsymbol{g}}|^{2}$ in a confined set of signals. Here, both $\hat{\boldsymbol{\rho}}$ and $\hat{\boldsymbol{g}}$ are vectors. Substituting $\hat{\boldsymbol{\rho}}=F \boldsymbol{\rho}$ as the discrete Fourier transform (DFT) of the time-domain signal $\rho$ by multiplying it with the DFT matrix $F$, the objective optimization function can be written as $E=\sum_{i=1}^{N}\left(\boldsymbol{\rho}^{T} F_{i}^{T} F_{i} \boldsymbol{\rho}-\hat{g}_{i}^{2}\right)^{2}$. Here $F_{i}$ is the $i$ th row of the DFT matrix $F$, and $\hat{g}_{i}$ is the $i$ th element of the measured $\hat{\boldsymbol{g}}$. The non-negative signal vector $\rho$ can be approximated by projection on a specific basis, for example, a basis of Gaussian functions. The approximated signal takes the form $\boldsymbol{\rho}=D \boldsymbol{x}$, where $D$ is the dictionary of the preset basis and $\boldsymbol{x}$ means the projection of the signal $\rho$ onto that basis. The dictionary can be a basis set of Gaussian functions, rectangular functions, a discrete point, or others dependent on the demand. We make an assumption that the electron current profile can be approximated by a linear combination of less than $s$ basis vectors defined by the dictionary $D$. With all these assumptions, the approximation of the signal $\rho$ can be obtained by minimizing the error function $E$.

For practical spectrum measurements, CTR spectra are unavoidably affected by noise introduced by detectors. If all the data with different noise levels in the spectra are given the same weight, the reconstructed beam current profiles will be seriously distorted. Therefore, the optimization function is amended as the weighted square of the distance between the reconstructed spectrum and the measured spectrum $E=\sum_{i=1}^{N} w_{i}\left(\boldsymbol{\rho}^{T} F_{i}^{T} F_{i} \boldsymbol{\rho}-\hat{g}_{i}{ }^{2}\right)^{2}$, where $w_{i}$ is the normalized weight introduced as an evaluation of the importance of each data. Here, $w_{i}=\sigma_{i}^{-2}\left(\sum_{i=1}^{n} \sigma_{i}^{-2}\right)^{-1}$, where $\sigma_{i}$ stands for the standard variance of spectrum data $i$. As a result, the mathematical formula of the phase retrieval problem constrained by the non-negativity, finite support, and sparsity priori can be expressed as

$$
\begin{aligned}
& \underset{x}{\arg \min } E=\sum_{i=1}^{N} w_{i}\left(\boldsymbol{x}^{T} D^{T} F_{i}^{T} F_{i} D \boldsymbol{x}-\hat{g}_{i}^{2}\right)^{2}, \\
& w_{i}=\frac{1}{\sigma_{i}^{2}\left(\sum_{i=1}^{n} \frac{1}{\sigma_{i}^{2}}\right)} \\
& \text { such that }\|\boldsymbol{x}\|_{0} \leq s, \\
& D \boldsymbol{x} \geq 0 .
\end{aligned}
$$

Here, $\|\boldsymbol{x}\|_{0}$ stands for the number of nonzero elements of $\boldsymbol{x}$, which should be less than the sparsity $s$. Note that the dictionary $D$ is chosen in the support so that the constraint of finite support can be automatically satisfied. For example, if the dictionary is chosen as the matrix based on Gaussian pulses with finite support, the reconstructed signal can be only the linear combination of those Gaussian pulses such that it also satisfies the support priority.

The flow chart of the WGESPAR algorithm is shown in Fig. 1. Let $\boldsymbol{n}$ be the index array of the nonzero elements of the vector $\boldsymbol{x}$, where $\boldsymbol{x}_{\boldsymbol{n}}$ means the nonzero elements of $\boldsymbol{x}$ such that the zero norm of $\boldsymbol{x}$ is equal to the length of the index array $\boldsymbol{n},\|\boldsymbol{x}\|_{0}=\operatorname{length}(\boldsymbol{n})$. The index array has a random initialization value $\boldsymbol{n}_{\mathbf{0}}$ with a length of $s$. At the $k$ th iteration, the optimization problem $\underset{x_{n_{k}}}{\arg \min E}=$ $\sum_{i=1}^{N} w_{i}\left(\boldsymbol{x}_{\boldsymbol{n}_{k}}{ }^{T} D_{\boldsymbol{n}_{k}}^{T} F_{i}^{T} F_{i} D_{\boldsymbol{n}_{k}} \boldsymbol{x}_{\boldsymbol{n}_{k}}-\hat{g}_{i}{ }^{2}\right)^{2}$ is solved by the nonlinear least-square trust-region algorithm [36], hence yielding $\boldsymbol{x}_{\boldsymbol{n}_{\boldsymbol{k}}}$ and $E_{\boldsymbol{n}_{\boldsymbol{k}}}$ with index array $\boldsymbol{n}_{k}$. Then, we swap the index of $M$ components of $\boldsymbol{x}_{\boldsymbol{n}_{k}}$ corresponding to the smallest absolute value and the $P$ negative maxima of $\nabla E(x)$. After this swap, the index array becomes $\boldsymbol{n}_{k}^{\prime}$, and $E_{\boldsymbol{n}_{k}^{\prime}}$ is yielded by solving the optimization function again. If the resultant $E_{\boldsymbol{n}_{k}^{\prime}}$ is smaller than $E_{\boldsymbol{n}_{k}}$, the $\boldsymbol{n}_{k}^{\prime}$ is reserved as the index array of the nonzero elements in the next loop $\boldsymbol{n}_{k+1}=\boldsymbol{n}_{k}^{\prime}$ until the value $E_{\boldsymbol{n}_{k}^{\prime}}$ of each swap is higher than the original $E_{\boldsymbol{n}_{k}}$. Then we accept $D \boldsymbol{x}_{\boldsymbol{n}_{k}}$ as the reconstructed beam current.

Three major factors, the sparsity $s$ and the parameters $M$ and $P$ during the swapping process, will influence the performance of the algorithm. The sparsity is determined by our prior knowledge of the beam currents. In order to greatly increase the computation efficiency, the current 


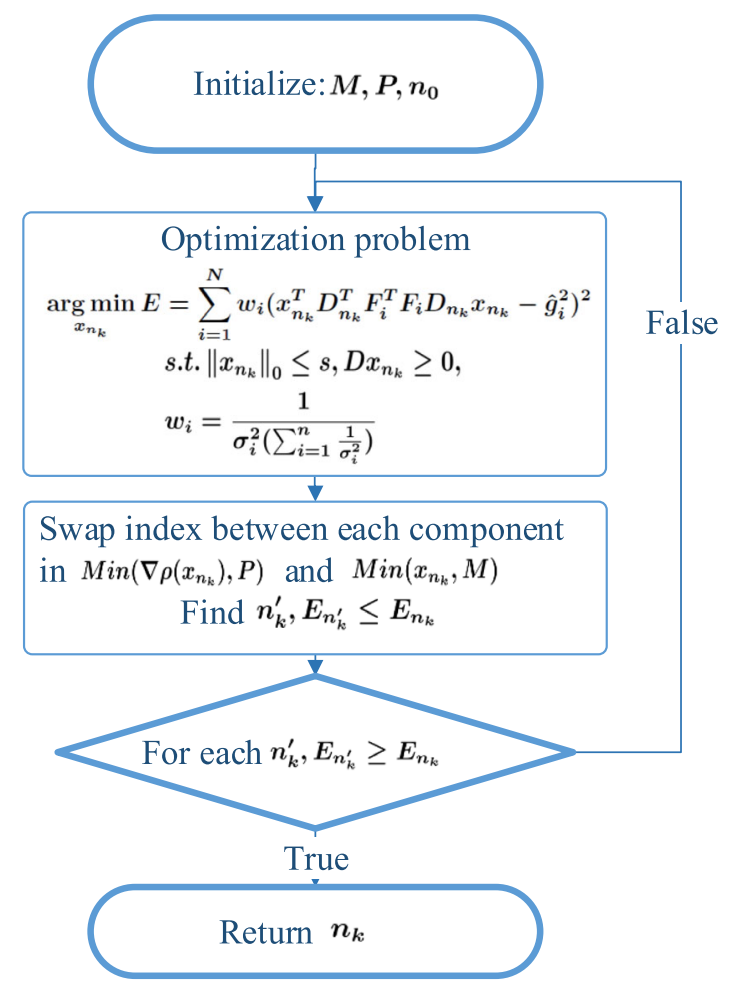

FIG. 1. Flow chart of the WGESPAR algorithm. $\operatorname{Min}(A, B)$ denotes the index array of the $B$ components of $A$ with the lowest value. The index array of the nonzero elements is initialized as $\boldsymbol{n}_{0}$, and the parameters of $M$ and $P$ are fixed during the reconstruction process.

profile is expected to be sparse on the chosen basis set. For a given basis, the sparsity can be increased for a better approximation of the real signal. The other two parameters $M$ and $P$ determine the convergence property of the algorithm. $M$ must be smaller than the sparsity, and $P$ must be smaller than the difference between the dictionary size and the sparsity. The convergence property of the WGESPAR algorithm can be improved when using a larger $P$ at the cost of computing time.

\section{B. Comparison of the WGESPAR, KK, and Bubblewrap algorithms for fs current reconstruction}

To compare the WGESPAR algorithm with the KK method and the Bubblewrap algorithm, we generate the original beam currents composed of multiple pulses, whose positions are randomly located in a support $[-30,30]$ fs and their durations are in the range of $[0.5,2]$ fs. The spectra of these original currents are sampled with a frequency step of $10 \mathrm{THz}$ based on the optical design of a midinfrared spectrometer and the resolution of a commercial midinfrared detector (for example, Pyrocam III from Ophir-Spiricon). In experiments, the CTR spectra are usually truncated outside a limited range. Here, the spectrum range is considered between 50 and $500 \mathrm{THz}$. These three phase retrieval algorithms are used to reconstruct the current profiles.
For the KK method, the CTR spectrum is generally extrapolated with the asymptotic fitting of the truncated spectrum [21]. For a multibunch current profile, the spectrum is modulated at a low frequency so that the extrapolation based on the quadratic fitting of few data will be inaccurate. Hence, we use Gaussian fitting of the spectrum between 50 and $70 \mathrm{THz}$ for extrapolation, which has been tested to have a better performance for the reconstruction of multibunch structures. For the Bubblewrap algorithm, there is no restriction in the frequency range below $50 \mathrm{THz}$, while for the WGESPAR algorithm, the basis is composed of Gaussian pulses for simplification. The pulse durations in the basis vary from 1 to $2 \mathrm{fs}$ with $0.2 \mathrm{fs}$ increments and from 0.5 to $1 \mathrm{fs}$ with $0.1 \mathrm{fs}$ increments. The locations of all Gaussian pulses in the basis are mapped uniformly with a specific interval in the support. Here, we choose an interval of $0.5 \mathrm{fs}$. The generated dictionary is based on Gaussian assumptions. During the reconstruction based on the WGESPAR algorithm, the calculation will be interrupted when either the distance between the measured and reconstructed spectrum is less than a preset threshold or the running cycles are larger than a preset number $N$. Here, we set $N=5$. For the WGESPAR algorithm, the parameters $M$ and $P$ are chosen to guarantee that the optimization algorithm converges to the global minimum while conserving the computational efficiency. The optimal values of $M$ and $P$ are dependent on the sparsity and complexity of the beam current. Here, we find that, for a sparsity less than 10 and a dictionary size of several hundred, $M=1$ or 2, $P=30$ is sufficient for convergence.

From Fig. 2, we can find that the asymptotic extrapolation of the CTR spectrum in the KK method is inaccurate when multiple pulses exist, leading to a false shape or elongated pulse length. The Bubblewrap algorithm presents a better performance in alleviating the elongated pulse length; however, sometimes it will bring ghost pulses or, on the contrary, miss pulses. With the assumption that the duration of each subbunch is less than $2 \mathrm{fs}$ in the dictionary, bunch trains with a support length of $60 \mathrm{fs}$ can be well reconstructed by the WGESPAR algorithm even without the frequency information below $50 \mathrm{THz}$. In practice, the influence of noise should be considered during the reconstruction of beam current profiles. Typical midinfrared pyroelectric cameras usually have a noise level of a few nanojoules, which is about 2 orders less than the expected CTR energy generated by $10 \mathrm{pC}$ electron charge. When $10 \%$ Gaussian noise is superimposed evenly on the spectrum in the wavelength domain, the temporal distribution can still be reconstructed [Figs. 2(b) and 2(d)]. The noise of the spectrum will increase as the frequency approaches zero due to variable substitution $\left|\frac{d I}{d \omega}\right|=\frac{\lambda^{2}}{2 \pi c}\left|\frac{d I}{d \lambda}\right|$. From the comparison in Figs. 2(b) and 2(d), the KK algorithm is more sensitive to noise and eventually fails to reconstruct the signal. Utilizing adaptive support selection to eliminate the influence of noise, the Bubblewrap algorithm shows a better performance than the 


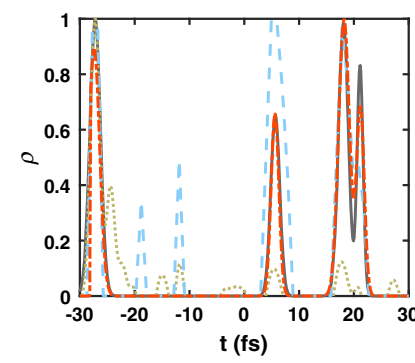

(a)

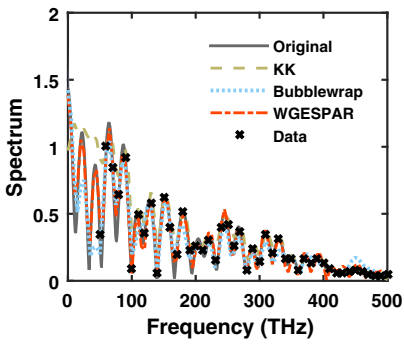

(c)

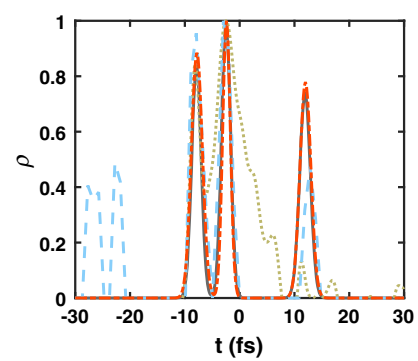

(b)

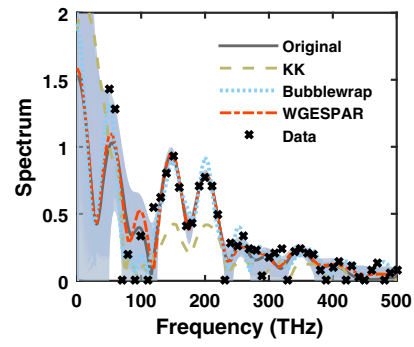

(d)

FIG. 2. The comparison of the WGESPAR, KK, and Bubblewrap algorithms for a current reconstruction with large support (a),(c) or noisy spectra (b),(d). The beam current profiles (a),(b) and the spectra (c),(d) are provided for the original current profile (gray line) and the results reconstructed by the WGESPAR algorithm (red dashed line), the KK algorithm (yellow dashed line), and the Bubblewrap algorithm (blue dotted line), respectively. The cross spots are sampled from the original spectrum, and the blue shading represents the uncertainty with $10 \%$ Gaussian noise in the wavelength domain.

KK algorithm by recognizing multibunch structure. However, the performance of the Bubblewrap algorithm is dependent on the parameter selection, such as the initial threshold and the decreasing rate of threshold for support recalculation. Since iterative algorithms tend to conform to all noisy data of the spectrum, misleading information will be unavoidably introduced. On the contrary, the WGESPAR algorithm only finds the solution in the preset group whose spectra are closest to the measured spectrum, therefore obtaining a better tolerance to noise.

To test the robustness of these three algorithms, the original currents are randomly generated, and three corresponding reconstruction results are obtained from the truncated spectra for 1000 times. The parameters of each algorithm are fixed during the robustness test. If the integrated difference between the original and reconstructed current profile is larger than $50 \%$ of the beam charge, we consider that the reconstruction fails. The recovery probability of each algorithm with respect to the number of superposed pulses (the defined sparsity in our algorithm) is shown in Fig. 3. The recovery probabilities of all the algorithms decrease with an increased pulse number due to the limited frequency sampling rate and the support selection with fixed parameters. Nevertheless, one can see that the WGESPAR

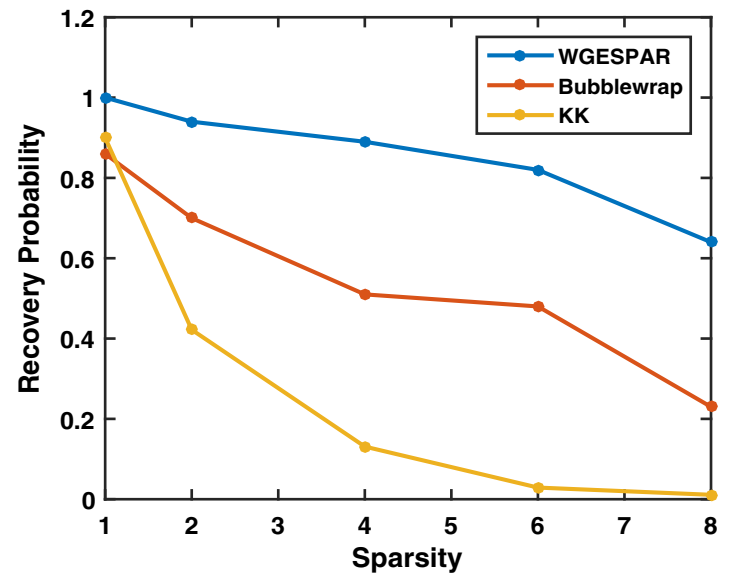

FIG. 3. Recovery probability of the KK method, the Bubblewrap algorithm, and the WGESPAR algorithm.

algorithm has the largest possibility to successfully reconstruct the current profiles.

Even with a basis set of Gaussian functions, the WGESPAR algorithm still shows good performance for special current profiles reconstruction such as multibunch spikes superposed on a long bunch or triangular shape as shown in Fig. 4. The reconstructed beam currents maintain main structural features such as the bunch train intervals or the ramp of the current transition. If other priories (such as

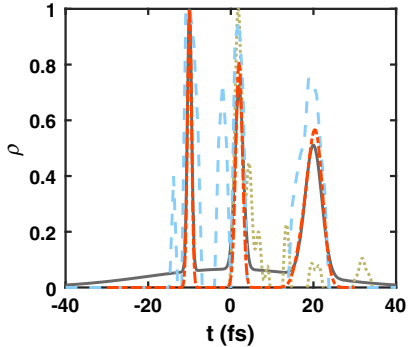

(a)

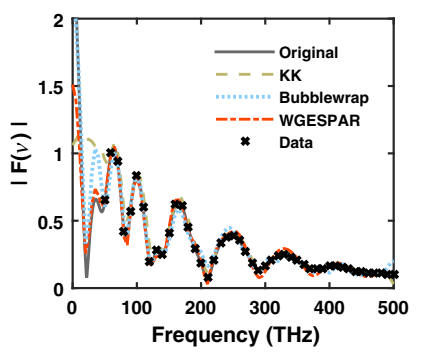

(c)

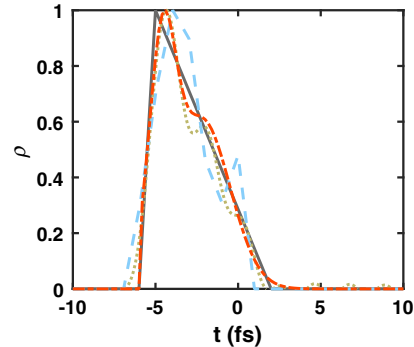

(b)

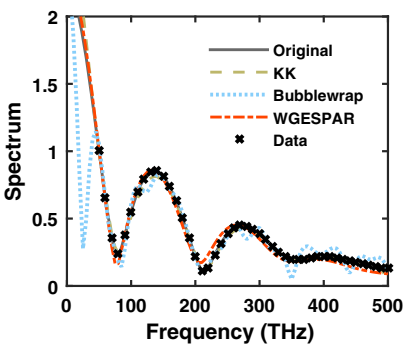

(d)
FIG. 4. Reconstructions of special beam current profiles: multibunch spikes superposed on a long bunch (a),(c) and triangular shape (b),(d). The beam current profiles (a),(b) and the spectra (c),(d) are provided for the original current profile (gray line) and the reconstruction result of the WGESPAR algorithm (red dashed line), the KK algorithm (yellow dashed line), and the Bubblewrap algorithm (blue dotted line), respectively. The cross spots are sampled from the original spectrum. 


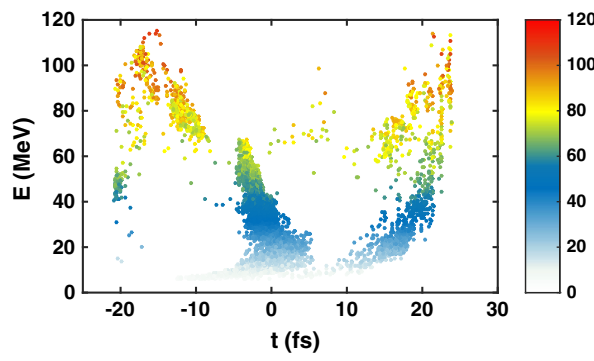

(a)

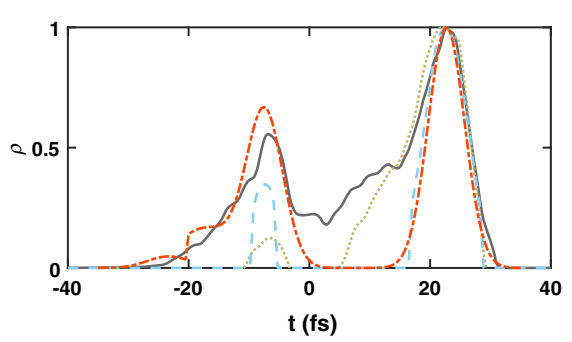

(b)

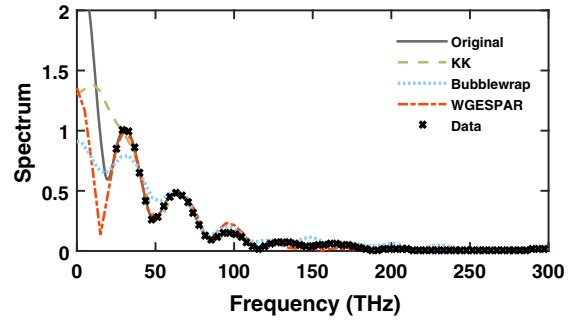

(c)

FIG. 5. The longitudinal phase space of the down-ramp injected electrons in the simulation (a) and the comparison of the simulated (gray line) and reconstructed beam current profiles by the WGESPAR algorithm (red dashed line), the KK algorithm (yellow dashed line), and the Bubblewrap algorithm (blue dotted line). The electron current profile (b) is reconstructed from the truncated simulated CTR spectrum (c) in the frequency range of 35-350 THz.

an approximation of the bunch shape) are provided, the reconstruction result will be much improved.

\section{Beam current reconstruction for synthetic simulation of PIC and Monte Carlo}

In the previous section, we demonstrate that the WGESPAR algorithm can effectively reconstruct complex current structures. To test the WGESPAR algorithm on the beam currents produced in laser plasma accelerators, we have performed 2D PIC simulations for down-ramp injected electron beams using OSIRIS. A 35 fs (rms), $400 \mathrm{~mJ}, 800 \mathrm{~nm}$ laser is focused at the front edge of a plasma with a diameter of $10 \mu \mathrm{m}$ (rms). The peak plasma density is set as $1.74 \times 10^{19} \mathrm{~cm}^{-3}$ with a sharp down ramp at $100 \mu \mathrm{m}$. Strong down-ramp injection of electrons with a two-pulse current structure has been observed in the simulation. The injected quasimonoenergetic electron bunch can be accelerated to $100 \mathrm{MeV}$ with an energy spread of $40 \%$ [Fig. 5(a)]. Using the accelerated electron bunches as the radiation source, the generation of CTR is simulated by the Monte Carlo method [21]. Because of the limited CTR collection aperture, electrons with an energy higher than $20 \mathrm{MeV}$ are considered as the CTR source. The current profiles are reconstructed from the spectra simulated by the Monte Carlo code with a sampling rate of $5 \mathrm{THz}$ from 35 to $350 \mathrm{THz}$.

We use the WGESPAR algorithm for phase retrieval with a support of $[-30,30]$ fs and the non-negativity constraint. A Gaussian basis with a pulse width varying from 1 to $4 \mathrm{fs}$, with an increment of $0.5 \mathrm{fs}$, is selected. We set the WGESPAR algorithm parameters as $M=1, P=30$, and $N=5$. The solution with the lowest sparsity is selected when the sparsity varies from 2 to 10 . Figure 5 shows the reconstructed result.

Figure 5 shows that the WGESPAR algorithm successfully reconstructs the feature of a two-peak current profile, which is better than the results reconstructed from the KK and Bubblewrap algorithms. The relative deviations of the current amplitude, beam intervals, and bunch length of each subbunch are less than 5\%.

\section{CONCLUSIONS}

It has been proven in previous studies that fs beam current profiles can be reconstructed from CTR spectra with a sufficient bandwidth. However, the obtained spectra in experiments are usually truncated, which will lead to inaccurate reconstructions for complex beam current profiles. To solve this problem, an optimization-based algorithm, WGESPAR, has been developed for the reconstruction of electron beam current profiles even with truncated noisy spectra. It has been found that the WGESPAR algorithm exhibits a better performance than the KK method and the Bubblewrap algorithm on multibunch profiles with a sparsity assumption. Using the synthetic simulation with PIC and Monte Carlo codes, the WGESPAR algorithm has been successfully used to reconstruct fs beam currents with complex temporal structures generated in laser-plasma accelerators. Moreover, this method has great potential in temporal diagnostics of bunch trains, thereby paving the way for applications of femtosecond electron beams with complex current profiles.

\section{ACKNOWLEDGMENTS}

We thank Dr. Wu Dai for discussing the KK method and providing the KK code for comparison. This work was supported by National Science Foundation of China Grants No. 11775125, No. 11425521, No. 11535006, No. 11375006, No. 11475101, and No. 11875175.

[1] Z. Zhang, L. Yan, Y. Du, Z. Zhou, X. Su, L. Zheng, D. Wang, Q. Tian, W. Wang, J. Shi, H. Chen, W. Huang, W. Gai, and C. Tang, Tunable High-Intensity Electron Bunch Train Production Based on Nonlinear Longitudinal Space Charge Oscillation, Phys. Rev. Lett. 116, 184801 (2016).

[2] C. Jing, A. Kanareykin, J. G. Power, M. Conde, Z. Yusof, P. Schoessow, and W. Gai, Observation of Enhanced Transformer Ratio in Collinear Wakefield Acceleration, Phys. Rev. Lett. 98, 144801 (2007).

[3] H. Ihee, V. A. Lobastov, U. M. Gomez, B. M. Goodson, R. Srinivasan, C.-Y. Ruan, and A. H. Zewail, Direct imaging of 
transient molecular structures with ultrafast diffraction, Science 291, 458 (2001).

[4] G. Sciaini, M. Harb, S. G. Kruglik, T. Payer, C. T. Hebeisen, F.-J. M. zu Heringdorf, M. Yamaguchi, M. Horn-von Hoegen, R. Ernstorfer, and R. D. Miller, Electronic acceleration of atomic motions and disordering in bismuth, Nature (London) 458, 56 (2009).

[5] K. Khrennikov, J. Wenz, A. Buck, J. Xu, M. Heigoldt, L. Veisz, and S. Karsch, Tunable All-Optical Quasimonochromatic Thomson X-Ray Source in the Nonlinear Regime, Phys. Rev. Lett. 114, 195003 (2015).

[6] S. Huang, Y. Ding, Y. Feng, E. Hemsing, Z. Huang, J. Krzywinski, A. A. Lutman, A. Marinelli, T. J. Maxwell, and D. Zhu, Generating Single-Spike Hard X-Ray Pulses with Nonlinear Bunch Compression in Free-Electron Lasers, Phys. Rev. Lett. 119, 154801 (2017).

[7] D. Alesini and F. Marcellini, rf deflector design of the CLIC test facility CTF3 delay loop and beam loading effect analysis, Phys. Rev. ST Accel. Beams 12, 031301 (2009).

[8] G. Berden, W. A. Gillespie, S. P. Jamison, E.-A. Knabbe, A. M. MacLeod, A. F. G. van der Meer, P. J. Phillips, H. Schlarb, B. Schmidt, P. Schmüser, and B. Steffen, Benchmarking of Electro-Optic Monitors for Femtosecond Electron Bunches, Phys. Rev. Lett. 99, 164801 (2007).

[9] H.-c. Lihn, P. Kung, C. Settakorn, H. Wiedemann, and D. Bocek, Measurement of subpicosecond electron pulses, Phys. Rev. E 53, 6413 (1996).

[10] M. Castellano, V. A. Verzilov, L. Catani, A. Cianchi, G. Orlandi, and M. Geitz, Measurements of coherent diffraction radiation and its application for bunch length diagnostics in particle accelerators, Phys. Rev. E 63, 056501 (2001).

[11] L. Zhao et al., Terahertz Streaking of Few-Femtosecond Relativistic Electron Beams, Phys. Rev. X 8, 021061 (2018).

[12] C. J. Zhang, J. F. Hua, Y. Wan, B. Guo, C.-H. Pai, Y. P. Wu, F. Li, H.-H. Chu, Y. Q. Gu, W. B. Mori, C. Joshi, J. Wang, and W. Lu, Temporal characterization of ultrashort linearly chirped electron bunches generated from a laser wakefield accelerator, Phys. Rev. Accel. Beams 19, 062802 (2016).

[13] H. Kotaki, K. Kawase, Y. Hayashi, M. Mori, M. Kando, J. K. Koga, and S. V. Bulanovt, Direct observation of the pulse width of an ultrashort electron beam, J. Phys. Soc. Jpn. 84, 074501 (2015).

[14] Z. Zhang, Y. Du, C. Tang, Y. Ding, and Z. Huang, Optical circular deflector with attosecond resolution for ultrashort electron beam, Phys. Rev. Accel. Beams 20, 050702 (2017).

[15] S. Casalbuoni, H. Schlarb, B. Schmidt, P. Schmüser, B. Steffen, and A. Winter, Numerical studies on the electro-optic detection of femtosecond electron bunches, Phys. Rev. ST Accel. Beams 11, 072802 (2008).

[16] P. Kung, H.-c. Lihn, H. Wiedemann, and D. Bocek, Generation and Measurement of 50-fs (rms) Electron Pulses, Phys. Rev. Lett. 73, 967 (1994).

[17] B. E. Billinghurst, J. C. Bergstrom, C. Baribeau, T. Batten, T. E. May, J. M. Vogt, and W. A. Wurtz, Longitudinal bunch dynamics study with coherent synchrotron radiation, Phys. Rev. Accel. Beams 19, 020704 (2016).

[18] O. Lundh et al., Few femtosecond, few kiloampere electron bunch produced by a laser-plasma accelerator, Nat. Phys. 7, 219 (2011).
[19] M. Islam et al., Near-threshold electron injection in the laser-plasma wakefield accelerator leading to femtosecond bunches, New J. Phys. 17, 093033 (2015).

[20] X. H. Lu, C. X. Tang, R. K. Li, H. To, G. Andonian, and P. Musumeci, Generation and measurement of velocity bunched ultrashort bunch of $\mathrm{pC}$ charge, Phys. Rev. ST Accel. Beams 18, 032802 (2015).

[21] R. Lai and A. Sievers, On using the coherent far IR radiation produced by a charged-particle bunch to determine its shape: I Analysis, Nucl. Instrum. Methods Phys. Res., Sect. A 397, 221 (1997).

[22] T. J. Maxwell, C. Behrens, Y. Ding, A. S. Fisher, J. Frisch, Z. Huang, and H. Loos, Coherent-Radiation Spectroscopy of Few-Femtosecond Electron Bunches Using a Middle-Infrared Prism Spectrometer, Phys. Rev. Lett. 111, 184801 (2013).

[23] D. Pelliccia and T. Sen, A two-step method for retrieving the longitudinal profile of an electron bunch from its coherent radiation, Nucl. Instrum. Methods Phys. Res., Sect. A 764, 206 (2014).

[24] S. I. Bajlekov, M. Heigoldt, A. Popp, J. Wenz, K. Khrennikov, S. Karsch, and S. M. Hooker, Longitudinal electron bunch profile reconstruction by performing phase retrieval on coherent transition radiation spectra, Phys. Rev. ST Accel. Beams 16, 040701 (2013).

[25] M. Heigoldt, A. Popp, K. Khrennikov, J. Wenz, S. W. Chou, S. Karsch, S. I. Bajlekov, S. M. Hooker, and B. Schmidt, Temporal evolution of longitudinal bunch profile in a laser wakefield accelerator, Phys. Rev. ST Accel. Beams 18, 121302 (2015).

[26] Y. Shechtman, A. Beck, and Y.C. Eldar, GESPAR: Efficient phase retrieval of sparse signals, IEEE Trans. Signal Process. 62, 928 (2014).

[27] P. Sidorenko, O. Kfir, Y. Shechtman, A. Fleischer, Y. C. Eldar, M. Segev, and O. Cohen, Sparsity-based superresolved coherent diffraction imaging of one-dimensional objects, Nat. Commun. 6, 8209 (2015).

[28] V. L. Ginzburg and I. M. Frank, On the transition radiation theory, Sov. Phys. JETP 16, 15 (1946).

[29] R. W. Gerchberg and W. O. Saxton, A practical algorithm for the determination of phase from image and diffraction plane pictures, Optik (Stuttgart) 35, 237 (1972).

[30] J. R. Fienup, Reconstruction of an object from the modulus of its Fourier transform, Opt. Lett. 3, 27 (1978).

[31] A. Walther, The question of phase retrieval in optics, J. Mod. Opt. 10, 41 (1963).

[32] R. Beinert, Non-negativity constraints in the onedimensional discrete-time phase retrieval problem, Inf. Inference 6, 213 (2017).

[33] H. Ohlsson and Y. C. Eldar, in Proceedings of the International Conference on Acoustics, Speech and Signal Processing (ICASSP) (IEEE, New York, 2014), pp. 1841-1845.

[34] E. Candès, Y. Eldar, T. Strohmer, and V. Voroninski, Phase retrieval via matrix completion, SIAM J. Imaging Sci. 6, 199 (2013).

[35] S. Mukherjee and C.S. Seelamantula, Fienup algorithm with sparsity constraints: Application to frequency-domain optical-coherence tomography, IEEE Trans. Signal Process. 62, 4659 (2014)

[36] P. Subramanian, Gauss-Newton methods for the complementarity problem, J. Optim. Theory Appl. 77, 467 (1993). 